\title{
Author Index Volume 43 (2015)
}

The issue number is given in front of the pagination

Adwan, L.I., see Subaiea, G.M. (2) 425-433

Aerts, T., see Dekker, A.D. (3) 871-891

Ager, R.R., see Dunn, H.C. (3) 893-903

Ahmed, A.H., see Subaiea, G.M. (2) 425-433

Aisen, P., see Liu-Seifert, H. (3) 949-955

Akatsu, H., see Uhm, K.-O. (1) 243-257

Alberoni, M., see Gironi, M. (4) 1199-1213

Alegret, M., see Hernández, I. (1) 325-334

Alkon, D.L., see Khan, T.K. (2) 491-509

Alkon, D.L., see Lucke-Wold, B.P. (3) 711-724

Allen, S.J., see Shoemark, D.K. (3) 725-738

Allué, J.A., see Pérez-Grijalba, V. (1) 47-56

Almeida, O.F.X., see Sotiropoulos, I. (3) 763-774

Almkvist, O., see Thordardottir, S. (4) 1393-1402

Almqvist, P., see Ferreira, D. (3) 1059-1072

Alonso, R., see Pisa, D. (2) 613-624

Amann, M., see Glodzik, L. (3) 939-947

Ambasta, R.K., see Kumar, P. (2) 341-361

Ambrose, C.T., A Therapeutic Approach for Senile Dementias: Neuroangiogenesis (1) 1-17

Ames, D., see Hollands, S. (2) 677-686

Ancín, I., see Cuesta, P. (1) 259-273

Andrade de Oliveira, A., M.T. Carthery-Goulart, P. Paulo de Magalhães Oliveira Júnior, D.C. Carrettiero, J.R. Sato and for the Alzheimer's Disease Neuroimaging Initiative, Defining Multivariate Normative Rules for Healthy Aging using Neuroimaging and Machine Learning: An Application to Alzheimer's Disease (1) 201-212

Ankarcrona, M., see Schreiner, B. (2) 369-374

Ansari, M.A., see Scheff, S.W. (3) 1073-1090

Arosio, B., see Serpente, M. (3) 757-761

Ascensión Zea-Sevilla, M., P. Bermejo-Velasco, R. Serrano-Heranz and M. Calero, Cerebral Autosomal Dominant Arteriopathy with Subcortical Infarcts and Leukoencephalopathy (CADASIL) associated with a Novel C82R Mutation in the NOTCH3 Gene (2) 363-367

Ash, E., see Oren, N. (1) 19-22

Aso, E.,A. Sánchez-Pla, E. Vegas-Lozano, R. Maldonado and I. Ferrer, Cannabis-Based Medicine Reduces
Multiple Pathological Processes in A $\beta P P / P S 1$ Mice (3) 977-991

Attia, J., see Muenchhoff, J. (4) 1355-1373

Auffray-Calvier, E., see Boutoleau-Bretonnière, C. (2) 625-630

Auriel, E., see Ni, J. (4) 1325-1330

Aurtenetxe, S., see Cuesta, P. (1) 259-273

Ayali, A., see Saad, Y. (3) 993-1006

Ayan, C., see Nascimento, C.M.C. (1) 81-91

Babb, J.S., see Glodzik, L. (3) 939-947

Baglietto-Vargas, D., see Dunn, H.C. (3) 893-903

Bagnoli, S., see Piaceri, I. (4) 1169-1173

Bajic, V., V. Mandusic, E. Stefanova, A. Bozovic, R. Davidovic, L. Zivkovic, A. Cabarkapa and B. Spremo-Potparevic, Skewed X-Chromosome Inactivation in Women Affected by Alzheimer's Disease (4) 1251-1259

Bajo, R., see Cuesta, P. (1) 259-273

Balsis, S. and D.A. Lowe, A Fresh Perspective Revealed by Powerful Trends in Alzheimer's Disease Research (1) 289-290

Barabash, A., see Cuesta, P. (1) 259-273

Barath, P., see Paholikova, K. (3) 915-926

Barbieri, F.A., see Simieli, L. (2) 435-441

Barmada, M.M., see Wang, X. (2) 649-655

Beattie, E., see Pachana, N.A. (3) 701-707

Becker, J.T., see Wang, X. (2) 649-655

Bedse, G., A. Romano, A.M. Lavecchia, T. Cassano and S. Gaetani, The Role of Endocannabinoid Signaling in the Molecular Mechanisms of Neurodegeneration in Alzheimer's Disease (4) $1115-1136$

Belfort, T., see Sousa, M.F.B. (3) 905-913

Benke, T., G. Sanin, A. Lechner, P. Dal-Bianco, G. Ransmayr, M. Uranüs, J. Marksteiner, M. Gaudig and R. Schmidt on behalf of the PRODEM Study Group, Predictors of Patient Dependence in Mildto-Moderate Alzheimer's Disease (2) 443-449

Ber, I.L., see Boutoleau-Bretonnière, C. (2) 625-630

Berezuk, C., see Ramirez, J. (2) 415-424 
Bermejo-Velasco, P., see Ascensión Zea-Sevilla, M. (2) 363-367

Bernasconi, M.P., see Santangelo, R. (4) 1429-1440

Bishnoi, R.J., R.F. Palmer and D.R. Royall, Vitamin D Binding Protein as a Serum Biomarker of Alzheimer's Disease (1) 37-45

Black, S.E., see McNeely, A.A. (3) 785-796

Black, S.E., see Ramirez, J. (2) 415-424

Blanthorn-Hazell, S., see Gough, M. (4) 1163-1168

Blaurock-Busch, E., see Calderón-Garcidueñas, L. (3) 1039-1058

Blennow, K., see Ferreira, D. (3) 1059-1072

Blennow, K., see Thordardottir, S. (4) 1393-1402

Boada, M., see Hernández, I. (1) 325-334

Boada, M., see Pérez-Grijalba, V. (1) 47-56

Bocti, C., see Castellano, C.-A. (4) 1343-1353

Boneschi, F.M., see Serpente, M. (3) 757-761

Bonoti, F., E. Tzouvaleka, K. Bonotis and F. Vlachos, Do Patients with Alzheimer's Disease Draw like Young Children? An Exploratory Study (4) 12851292

Bonotis, K., see Bonoti, F. (4) 1285-1292

Bonsi, R., see Serpente, M. (3) 757-761

Borawska, R., see Mroczko, B. (3) 1031-1037

Borgiani, B., see Gironi, M. (4) 1199-1213

Borrie, M., see Montero-Odasso, M. (1) 193-199

Boutoleau-Bretonnière, C., A. Camuzat, I.L. Ber, K. Bouya-Ahmed, R. Guerreiro, A.-L. Deruet, C. Evrard, J. Bras, E. Lamy, E. Auffray-Calvier, A. Pallardy, J. Hardy, A. Brice, P. Derkinderen and M. Vercelletto, A Phenotype of Atypical Apraxia of Speech in a Family Carrying SQSTM1 Mutation (2) 625-630

Bouya-Ahmed, K., see Boutoleau-Bretonnière, C. (2) 625-630

Bozovic, A., see Bajic, V. (4) 1251-1259

Bozzelli, P.L., see Graybeal, J.J. (3) 823-834

Bras, J., see Boutoleau-Bretonnière, C. (2) 625-630

Brice, A., see Boutoleau-Bretonnière, C. (2) 625-630

Brodaty, H., see Muenchhoff, J. (4) 1355-1373

Buckley, R., see Hollands, S. (2) 677-686

Buscema, M., see Gironi, M. (4) 1199-1213

Busch, Y., see Calderón-Garcidueñas, L. (3) 1039-1058

Byberg, L., see Olsson, E. (1) 109-119

Byrjalsen, I., see Henriksen, K. (4) 1331-1341

Cabarkapa, A., see Bajic, V. (4) 1251-1259

Cabranes, J.A., see Cuesta, P. (1) 259-273

Cacciatore, F., see Maderna, E. (2) 375-379

Calderón-Garcidueñas, L., A. Vojdani, E. BlaurockBusch, Y. Busch, A. Friedle, M. Franco-Lira, P. Sarathi-Mukherjee, X. Martínez-Aguirre, S.-B. Park, R. Torres-Jardón and A. D’Angiulli, Air
Pollution and Children: Neural and Tight Junction Antibodies and Combustion Metals, the Role of Barrier Breakdown and Brain Immunity in Neurodegeneration (3) 1039-1058

Calero, M., see Ascensión Zea-Sevilla, M. (2) 363-367 Campbell, S.N., C. Zhang, L. Monte, A.D. Roe, K.C. Rice, Y. Taché, E. Masliah and R.A. Rissman, Increased Tau Phosphorylation and Aggregation in the Hippocampus of Mice Overexpressing Corticotropin-Releasing Factor (3) 967-976

Camuzat, A., see Boutoleau-Bretonnière, C. (2) 625630

Cao, L., see Li, J.-c. (2) 535-548

Cappa, S.F., see Cerami, C. (2) 385-395

Carmona, H., see Gifford, K.A. (1) 121-132

Carrasco, L., see Pisa, D. (2) 613-624

Carrettiero, D.C., see Andrade de Oliveira, A. (1) 201212

Carthery-Goulart, M.T., see Andrade de Oliveira, A. (1) 201-212

Casabona, D., see Pérez-Grijalba, V. (1) 47-56

Cassano, T., see Bedse, G. (4) 1115-1136

Castellano, C.-A., S. Nugent, N. Paquet, S. Tremblay, C. Bocti, G. Lacombe, H. Imbeault, É. Turcotte, T. Fulop and S.C. Cunnane, Lower Brain ${ }^{18}$ F-Fluorodeoxyglucose Uptake But Normal ${ }^{11} \mathrm{C}$-Acetoacetate Metabolism in Mild Alzheimer's Disease Dementia (4) 1343-1353

Catania, M., see Maderna, E. (2) 375-379

Cattaneo, C., see Maderna, E. (2) 375-379

Cederholm, T., see Olsson, E. (1) 109-119

Cerami, C., C. Crespi, P.A.D. Rosa, A. Dodich, A. Marcone, G. Magnani, E. Coppi, A. Falini, S.F. Cappa and D. Perani, Brain Changes within the Visuo-Spatial Attentional Network in Posterior Cortical Atrophy (2) 385-395

Cespón, J., S. Galdo-Álvarez, A.X. Pereiro and F. Díaz, Differences between Mild Cognitive Impairment Subtypes as Indicated by Event-Related Potential Correlates of Cognitive and Motor Processes in a Simon Task (2) 631-647

Chai, D.-M., see Du, L.-L. (3) 775-784

Chang, Y.J., see Song, H. (3) 797-807

Chen, H., see Li, J.-c. (2) 535-548

Chen, J., H. Shu, Z. Wang, D. Liu, Y. Shi, X. Zhang and Z. Zhang, The Interaction of APOE Genotype by Age in Amnestic Mild Cognitive Impairment: A Voxel-Based Morphometric Study (2) 657-668

Chen, J., see Wang, J. (2) 465-477

Chen, J.X., see Wang, Y. (2) 451-463

Chen, T., R. Hou, C. Li, C. Wu and S. Xu, MPTP/MPP+ Suppresses Activation of Protein C in Parkinson's Disease (1) 133-142 
Chen, W., see Zhang, B. (2) 687-697

Chen, Y.-W., see Lucke-Wold, B.P. (3) 711-724

Cheng, D., see Dunn, H.C. (3) 893-903

Cheng, N., see Peng, L. (1) 227-242

Cheong, H.-K., see Ye, B.S. (1) 143-152

Chiam, J.T.W., R.J.B. Dobson, S.J. Kiddle and M. Sattlecker, Are Blood-Based Protein Biomarkers for Alzheimer's Disease also Involved in Other Brain Disorders? A Systematic Review (1) 303314

Chin, J., see Ye, B.S. (1) 143-152

Choi, E.-K., see Jung, C.-G. (4) 1215-1228

Choi, E.-K., see Uhm, K.-O. (1) 243-257

Choi, S.H., see Ye, B.S. (1) 143-152

Christiansen, C., see Henriksen, K. (4) 1331-1341

Chukkapalli, S., see Poole, S. (1) 67-80

Cichocki, A., see Gallego-Jutglà, E. (4) 1175-1184

Ciobanu, I., see Hagl, S. (3) 927-938

Cioffi, S.M.G., see Serpente, M. (3) 757-761

Clerici, F., see Serpente, M. (3) 757-761

Comi, G., see Gironi, M. (4) 1199-1213

Comi, G., see Santangelo, R. (4) 1429-1440

Cominetti, M.R., see Nascimento, C.M.C. (1) 81-91

Coppi, E., see Cerami, C. (2) 385-395

Coppi, E., see Santangelo, R. (4) 1429-1440

Coppus, A.M.W., see Dekker, A.D. (3) 871-891

Cortes, E.P., see Seifan, A. (1) 315-324

Costa, P., see Sotiropoulos, I. (3) 763-774

Cox, D.N., see Graybeal, J.J. (3) 823-834

Crean, S., see Poole, S. (1) 67-80

Cremer, B., see Perrar, K.M. (2) 397-413

Crespi, C., see Cerami, C. (2) 385-395

Cribbs, D.H., see Dunn, H.C. (3) 893-903

Cruces, J., see Maté, I. (1) 213-226

Crutch, S.J., see Shakespeare, T.J. (2) 381-384

Cubinkova, V., see Lejavova, K. (4) 1157-1161

Cuesta, P., A. Barabash, S. Aurtenetxe, P. Garcés, M.E. López, R. Bajo, M. Llanero-Luque, I. Ancín, J.A. Cabranes, A. Marcos, M. Sancho, A. Nakamura, F. Maestú and A. Fernandez, Source Analysis of Spontaneous Magnetoencephalograpic Activity in Healthy Aging and Mild Cognitive Impairment: Influence of Apolipoprotein E Polymorphism (1) 259-273

Cui, L.-q., see Tan, J.-p. (4) 1403-1412

Cummings, J., see Liu-Seifert, H. (3) 949-955

Cunnane, S.C., see Castellano, C.-A. (4) 1343-1353

Cursano, C., see Gironi, M. (4) 1199-1213

D’Angiulli, A., see Calderón-Garcidueñas, L. (3) 10391058

Dai, H., see Zhao, X. (2) 549-563

Dal-Bianco, P., see Benke, T. (2) 443-449
Dam, D.V., see Dekker, A.D. (3) 871-891

Darreh-Shori, T., see Ferreira, D. (3) 1059-1072

Dauwels, J., see Gallego-Jutglà, E. (4) 1175-1184

Davidovic, R., see Bajic, V. (4) 1251-1259

De Deyn, P.P., see Dekker, A.D. (3) 871-891

De la Fuente, M., see Maté, I. (1) 213-226

de Lucia, N., see Grossi, D. (3) 849-855

Dekker, A.D., A.M.W. Coppus, Y. Vermeiren, T. Aerts, C.M. van Duijn, B.P. Kremer, P.J.W. Naudé, D.V. Dam and P.P. De Deyn, Serum MHPG Strongly Predicts Conversion to Alzheimer's Disease in Behaviorally Characterized Subjects with Down Syndrome (3) 871-891

DeKosky, S.T., see Wang, X. (2) 649-655

Deloncle, R. and O. Guillard, Is Brain Copper Deficiency in Alzheimer's, Lewy Body, and Creutzfeldt Jakob Diseases the Common Key for a Free Radical Mechanism and Oxidative StressInduced Damage? (4) 1149-1156

Demirci, F.Y., see Wang, X. (2) 649-655

Derkinderen, P., see Boutoleau-Bretonnière, C. (2) 625630

Deruet, A.-L., see Boutoleau-Bretonnière, C. (2) 625630

Díaz, F., see Cespón, J. (2) 631-647

Díaz, M., N. Fabelo, V. Martín, I. Ferrer, T. Gómez and R. Marín, Biophysical Alterations in Lipid Rafts from Human Cerebral Cortex Associate with Increased BACE1/A $\beta P P$ Interaction in Early Stages of Alzheimer's Disease (4) 1185-1198

DiBernardo, A., see Tryputsen,V. (3) 809-821

Dobson, R.J.B., see Chiam, J.T.W. (1) 303-314

Dodich, A., see Cerami, C. (2) 385-395

Domingues, M.R.M., see Monteiro-Cardoso, V.F. (4) 1375-1392

Dourado, M.C.N., see Sousa, M.F.B. (3) 905-913

Dricot, L., see Ivanoiu, A. (3) 835-847

Du, L.-L., D.-M. Chai, L.-N. Zhao, X.-H. Li, F.-C.Zhang, H.-B. Zhang, L.-B. Liu, K. Wu, R. Liu, J.-Z. Wang and X.-W. Zhou, AMPK Activation Ameliorates Alzheimer's Disease-Like Pathology and Spatial Memory Impairment in a Streptozotocin-Induced Alzheimer's Disease Model in Rats (3) 775-784

Du, L.-S., see Yu, D. (1) 291-302

Du, W., see Tan, J.-p. (4) 1403-1412

Duncan, F.J., see Naughton, B.J. (1) 93-108

Duncan, M., see Muenchhoff, J. (4) 1355-1373

Dunn, H.C., R.R. Ager, D. Baglietto-Vargas, D. Cheng, M. Kitazawa, D.H. Cribbs and R. Medeiros, Restoration of Lipoxin $\mathrm{A}_{4}$ Signaling Reduces Alzheimer's Disease-Like Pathology in the 3xTgAD Mouse Model (3) 893-903

Dupuy, C., see Villars, H. (1) 167-176 
Eckert, G.P., see Hagl, S. (3) 927-938

Eichler, T., D. Wucherer, J.R. Thyrian, I. Kilimann, J. Hertel, B. Michalowsky, S. Teipel and W. Hoffmann, Antipsychotic Drug Treatment in Ambulatory Dementia Care: Prevalence and Correlates (4) 1303-1311

Eisenmann, Y., see Perrar, K.M. (2) 397-413

Ellis, K.A., see Hollands, S. (2) 677-686

Emmerzaal, T.L., A.J. Kiliaan and D.R. Gustafson, 2003-2013: A Decade of Body Mass Index, Alzheimer's Disease, and Dementia (3) 739-755

Eriksdotter, M. see Ferreira, D. (3) 1059-1072

Eriksdotter, M., see Thordardottir, S. (4) 1393-1402

Eslick, G.D., see Maheshwari, P. (3) 957-966

Espinosa, A., see Hernández, I. (1) 325-334

Evrard, C., see Boutoleau-Bretonnière, C. (2) 625-630

Eyjolfsdottir, H., see Ferreira, D. (3) 1059-1072

Fabelo, N., see Díaz, M. (4) 1185-1198

Falini, A., see Cerami, C. (2) 385-395

Fang, D., see Wang, Y. (2) 451-463

Farina, E., see Gironi, M. (4) 1199-1213

Faure, C., see Takizawa, C. (4) 1271-1284

Fede, G.D., see Maderna, E. (2) 375-379

Fenoglio, C., see Serpente, M. (3) 757-761

Fernandez, A., see Cuesta, P. (1) 259-273

Ferrari, L., see Santangelo, R. (4) 1429-1440

Ferreira, D., E. Westman, H. Eyjolfsdottir, P. Almqvist, G. Lind, B. Linderoth, Å. Seiger, K. Blennow, A. Karami, T. Darreh-Shori, M. Wiberg, A. Simmons, L.-O. Wahlund, L. Wahlberg, M. Eriksdotter and for the Alzheimer's Disease Neuroimaging Initiative, Brain Changes in Alzheimer's Disease Patients with Implanted Encapsulated Cells Releasing Nerve Growth Factor (3) 1059-1072

Ferreira, D., see Thordardottir, S. (4) 1393-1402

Ferreiro, E., see Monteiro-Cardoso, V.F. (4) 1375-1392 Ferrer, I., see Aso, E. (3) 977-991

Ferrer, I., see Díaz, M. (4) 1185-1198

Filipcik, P., see Lejavova, K. (4) 1157-1161

Flinn, J.M., see Graybeal, J.J. (3) 823-834

Fotiadis, P., see Ni, J. (4) 1325-1330

Foxe, D., see Shakespeare, T.J. (2) 381-384

Franceschi, M., see Serpente, M. (3) 757-761

Franco-Lira, M., see Calderón-Garcidueñas, L. (3) 1039-1058

Friede, T., see Schmidt, C. (4) 1229-1236

Friedle, A., see Calderón-Garcidueñas, L. (3) 10391058

Fu, H., see Naughton, B.J. (1) 93-108

Fu, Y.H., J.-H.T. Hsiao, G. Paxinos, G.M. Halliday and W.S. Kim, ABCA5 Regulates Amyloid- $\beta$ Peptide
Production and is Associated with Alzheimer's Disease Neuropathology (3) 857-869

Fulop, T., see Castellano, C.-A. (4) 1343-1353

Furlan, R., see Gironi, M. (4) 1199-1213

Gaetani, S., see Bedse, G. (4) 1115-1136

Galasko, D., see Mackin, R.S. (3) 1007-1016

Galdo-Álvarez, S., see Cespón, J. (2) 631-647

Galimberti, D., see Serpente, M. (3) 757-761

Gallego-Jutglà, E., J. Solé-Casals, F.-B. Vialatte, J. Dauwels and A. Cichocki, A Theta-Band EEG Based Index for Early Diagnosis of Alzheimer's Disease (4) 1175-1184

Gao, F., see McNeely, A.A. (3) 785-796

Gao, F., see Ramirez, J. (2) 415-424

Gao, J., see Tan, J.-p. (4) 1403-1412

Gao, X., see Wang, D. (2) 519-534

Garcés, P., see Cuesta, P. (1) 259-273

Garner, B., see Zhao, H. (3) 1017-1030

Garuffi, M., see Nascimento, C.M.C. (1) 81-91

Gass, A., see Glodzik, L. (3) 939-947

Gaudig, M., see Benke, T. (2) 443-449

Gazagnes, M.-D. see Safouris, A. (1) 23-27

Gellerfors, P., see Tucker, S. (2) 575-588

Gerlach, N., see Schmidt, C. (4) 1229-1236

Gherib, K., see Schmidt, C. (4) 1229-1236

Giaccone, G., see Maderna, E. (2) 375-379

Gifford, K.A., D. Liu, H. Carmona, Z. Lu, R. Romano, Y. Tripodis, B. Martin, N. Kowall and A.L. Jefferson, Inclusion of an Informant Yields Strong Associations between Cognitive Complaint and Longitudinal Cognitive Outcomes in NonDemented Elders (1) 121-132

Giladi, N., see Oren, N. (1) 19-22

Gilis, N., see Ivanoiu, A. (3) 835-847

Giménez-Llort, L., see Maté, I. (1) 213-226

Gironi, M., B. Borgiani, E. Farina, E. Mariani, C. Cursano, M. Alberoni, R. Nemni, G. Comi, M. Buscema, R. Furlan and E. Grossi, A Global Immune Deficit in Alzheimer's Disease and Mild Cognitive Impairment Disclosed by a Novel Data Mining Process (4) 1199-1213

Glodzik, L., M. Sollberger, A. Gass, A. Gokhale, H. Rusinek, J.S. Babb, J.G. Hirsch, M. Amann, A.U. Monsch and O. Gonen, Global N-Acetylaspartate in Normal Subjects, Mild Cognitive Impairment and Alzheimer's Disease Patients (3) 939-947

Gobbi, L.T.B., see Simieli, L. (2) 435-441

Gokhale, A., see Glodzik, L. (3) 939-947

Gómez, T., see Díaz, M. (4) 1185-1198

Gonen, O., see Glodzik, L. (3) 939-947

Gopaul, K., see Montero-Odasso, M. (1) 193-199 
Gough, M., S. Blanthorn-Hazell and E.T. Parkin, The Histidine Composition of the Amyloid- $\beta$ Domain, but not the E1 Copper Binding Domain, Modulates $\beta$-Secretase Processing of Amyloid- $\beta$ Protein Precursor in Alzheimer's Disease (4) 1163-1168

Graff, C., see Thordardottir, S. (4) 1393-1402

Grandin, C., see Ivanoiu, A. (3) 835-847

Graybeal, J.J., P.L. Bozzelli, L.L. Graybeal, C.M. Groeber, P.E. McKnight, D.N. Cox and J.M. Flinn, Human ApoE $\varepsilon 4$ Alters Circadian Rhythm Activity, IL-1 $\beta$, and GFAP in CRND8 Mice (3) 823-834

Graybeal, L.L., see Graybeal, J.J. (3) 823-834

Greenberg, S.M., see Ni, J. (4) 1325-1330

Grewal, R., see Hagl, S. (3) 927-938

Groblewska, M., see Mroczko, B. (3) 1031-1037

Groeber, C.M., see Graybeal, J.J. (3) 823-834

Grossi, D., N. de Lucia and L. Trojano, Closing-in is related to Apathy in Alzheimer's Disease Patients (3) $849-855$

Grossi, E., see Gironi, M. (4) 1199-1213

Gu, Q.-1., see Li, J.-c. (2) 535-548

Guerreiro, R., see Boutoleau-Bretonnière, C. (2) 625630

Guillard, O., see Deloncle, R. (4) 1149-1156

Gurol, E.M., see Ni, J. (4) 1325-1330

Gustafson, D.R., see Emmerzaal, T.L. (3) 739-755

Haapasalo, A., see Martiskainen, H. (2) 565-573

Haghighi, M., A. Smith, D. Morgan, B. Small, S. Huang and for the Alzheimer's Disease Neuroimaging Initiative (ADNI), Identifying Cost-Effective Predictive Rules of Amyloid- $\beta$ Level by Integrating Neuropsychological Tests and PlasmaBased Markers (4) 1261-1270

Hagl, S., R. Grewal, I. Ciobanu, A. Helal, M.T. Khayyal, W.E. Muller and G.P. Eckert, Rice Bran Extract Compensates Mitochondrial Dysfunction in a Cellular Model of Early Alzheimer's Disease (3) $927-938$

Hall, A., see Martiskainen, H. (2) 565-573

Halliday, G.M., see Fu, Y.H. (3) 857-869

Hambye, A.-S., see Safouris, A. (1) 23-27

Han, A.H., see Hradek, A.C. (1) 57-65

Han, B., see Liu-Seifert, H. (3) 949-955

Han, L., see Li, J.-c. (2) 535-548

Han, S.-H., see Ye, B.S. (1) 143-152

Hanseeuw, B., see Ivanoiu, A. (3) 835-847

Hardy, J., see Boutoleau-Bretonnière, C. (2) 625-630

Harrington, K., see Hollands, S. (2) 677-686

He, H., see Peng, L. (1) 227-242

He, R., see Wang, D. (2) 519-534
He, R.-r., see Li, J.-c. (2) 535-548

He, X.-d., see Li, J.-c. (2) 535-548

He, X.-J., see Yu, D. (1) 291-302

Hedskog, L., see Schreiner, B. (2) 369-374

Hegedusova, N., see Lejavova, K. (4) 1157-1161

Helal, A., see Hagl, S. (3) 927-938

Helisalmi, S., see Martiskainen, H. (2) 565-573

Hendler, T., see Oren, N. (1) 19-22

Henriksen, K., I. Byrjalsen, C. Christiansen and M.A. Karsdal, Relationship between Serum Levels of Tau Fragments and Clinical Progression of Alzheimer's Disease (4) 1331-1341

Hernández, I., M. Rosende-Roca, M. Alegret, A. Mauleón, A. Espinosa, L. Vargas, O. SotolongoGrau, L. Tárraga, M. Boada and A. Ruiz, Association of TMEM106B rs1990622 Marker and Frontotemporal Dementia: Evidence for a Recessive Effect and Meta-Analysis (1) 325-334

Hertel, J., see Eichler, T. (4) 1303-1311

Herukka, S.-K., see Martiskainen, H. (2) 565-573

Hida, H., see Jung, C.-G. (4) 1215-1228

Hida, H., see Uhm, K.-O. (1) 243-257

Hiltunen, M., see Martiskainen, H. (2) 565-573

Hirsch, J.G., see Glodzik, L. (3) 939-947

Hjorth, E., see Zhu, M. (4) 1237-1250

Hoang, V.-H., see Song, H. (3) 797-807

Hodges, J., see Shakespeare, T.J. (2) 381-384

Hoffmann, W., see Eichler, T. (4) 1303-1311

Hogervorst, E., see Xu, X. (2) 669-675

Holder, D.J., see Zhao, X. (2) 549-563

Hollands, S., Y.Y. Lim, R. Buckley, R.H. Pietrzak, P.J. Snyder, D. Ames, K.A. Ellis, K. Harrington, N. Lautenschlager, R.N. Martins, C.L. Masters, V.L. Villemagne, C.C. Rowe, P. Maruff and for the AIBL Research Group, Amyloid- $\beta$ Related Memory Decline is not Associated with Subjective or Informant Rated Cognitive Impairment in Healthy Adults (2) 677-686

Hong, C.H., see Ye, B.S. (1) 143-152

Hongpaisan, J., see Khan, T.K. (2) 491-509

Honig, L.S., see Seifan, A. (1) 315-324

Horike, H., see Jung, C.-G. (4) 1215-1228

Horvathova, L., see Lejavova, K. (4) 1157-1161

Hou, R., see Chen, T. (1) 133-142

Hradek, A.C., H.-P. Lee, S.L. Siedlak, S.L. Torres, W. Jung, A.H. Han and H.-g. Lee, Distinct Chronology of Neuronal Cell Cycle Re-Entry and Tau Pathology in the 3xTg-AD Mouse Model and Alzheimer's Disease Patients (1) 57-65

Hsiao, J.-H.T., see Fu, Y.H. (3) 857-869

Huang, G., see Ma, F. (4) 1441-1449

Huang, H., see Ma, Q. (4) 1413-1427 
Huang, R., see Li, J.-c. (2) 535-548

Huang, S., see Haghighi, M. (4) 1261-1270

Huang, X., see Ma, Q. (4) 1413-1427

Huber, J.D., see Lucke-Wold, B.P. (3) 711-724

Hui, Y., see Wang, D. (2) 519-534

Huovinen, J., see Martiskainen, H. (2) 565-573

Ikonomovic, M.D., see Scheff, S.W. (3) 1073-1090

Imbeault, H., see Castellano, C.-A. (4) 1343-1353

Insel, P., see Mackin, R.S. (3) 1007-1016

Inzitari, D., see Salvadori, E. (4) 1313-1323

Ishida, A., see Jung, C.-G. (4) 1215-1228

Islam, A., see Montero-Odasso, M. (1) 193-199

Ivanoiu, A., L. Dricot, N. Gilis, C. Grandin, R. Lhommel,

L. Quenon and B. Hanseeuw, Classification of Non-Demented Patients Attending a Memory Clinic using the New Diagnostic Criteria for Alzheimer's Disease with Disease-Related Biomarkers (3) 835-847

Jefferson, A.L., see Gifford, K.A. (1) 121-132

Jeong, J.H., see Ye, B.S. (1) 143-152

Jha, N.K., see Kumar, P. (2) 341-361

Jha, S.K., see Kumar, P. (2) 341-361

Jiang, T., see Tan, M.-S. (2) 589-603

Jin, J., see Wang, D. (2) 519-534

Juang, C., see Pachana, N.A. (3) 701-707

Juarranz, A., see Pisa, D. (2) 613-624

Jung, C.-G., K.-O. Uhm, H. Horike, M.-J. Kim, S. Misumi, A. Ishida, Y. Ueda, E.-K. Choi, Y.-S.

Kim, M. Michikawa and H. Hida, Auraptene Increases the Production of Amyloid- $\beta$ via c-Jun $\mathrm{N}$-Terminal Kinase-Dependent Activation of $\gamma$-Secretase (4) 1215-1228

Jung, C.-G., see Uhm, K.-O. (1) 243-257

Jung, W., see Hradek, A.C. (1) 57-65

Kamboh, M.I., see Wang, X. (2) 649-655

Karami, A., see Ferreira, D. (3) 1059-1072

Karlström, B., see Olsson, E. (1) 109-119

Karsdal, M.A., see Henriksen, K. (4) 1331-1341

Kawaguchi, M., see Uhm, K.-O. (1) 243-257

Keil, B., see Ni, J. (4) 1325-1330

Kerr, D.S., see Nascimento, C.M.C. (1) 81-91

Kesavalu, L., see Poole, S. (1) 67-80

Khan, T.K., A. Sen, J. Hongpaisan, C.S. Lim, T.J. Nelson and D.L. Alkon, PKCe Deficits in Alzheimer's Disease Brains and Skin Fibroblasts (2) 491-509

Khayyal, M.T., see Hagl, S. (3) 927-938

Kiddle, S.J., see Chiam, J.T.W. (1) 303-314

Kilander, L., see Olsson, E. (1) 109-119
Kiliaan, A.J., see Emmerzaal, T.L. (3) 739-755

Kilimann, I., see Eichler, T. (4) 1303-1311

Kim, E.-J., see Ye, B.S. (1) 143-152

Kim, M.-J., see Jung, C.-G. (4) 1215-1228

Kim, M.-J., see Uhm, K.-O. (1) 243-257

Kim, S., see Ye, B.S. (1) 143-152

Kim, S.Y., see Ye, B.S. (1) 143-152

Kim, W.S., see Fu, Y.H. (3) 857-869

Kim, Y.-S., see Jung, C.-G. (4) 1215-1228

Kim, Y.-S., see Uhm, K.-O. (1) 243-257

Kimura, T., see Sotiropoulos, I. (3) 763-774

Kiss, A., see McNeely, A.A. (3) 785-796

Kitazawa, M., see Dunn, H.C. (3) 893-903

Knight, B.G., see Pachana, N.A. (3) 701-707

Koivisto, A.M., see Martiskainen, H. (2) 565-573

Kornhuber, J., see Lewczuk, P. (1) 183-191

Kornhuber, J., see Mroczko, B. (3) 1031-1037

Kovacech, B., see Paholikova, K. (3) 915-926

Kowall, N., see Gifford, K.A. (1) 121-132

Kremer, B.P., see Dekker, A.D. (3) 871-891

$\mathrm{Ku}$, B.D., see Ye, B.S. (1) 143-152

Kumar, P., N.K. Jha, S.K. Jha, K. Ramani and R.K. Ambasta, Tau Phosphorylation, Molecular Chaperones, and Ubiquitin E3 Ligase: Clinical Relevance in Alzheimer's Disease (2) 341-361

Kurihara, H., see Li, J.-c. (2) 535-548

Kurki, M., see Martiskainen, H. (2) 565-573

Kurkinen, K.M.A., see Martiskainen, H. (2) 565-573

Kvetnansky, R., see Lejavova, K. (4) 1157-1161

Lacombe, G., see Castellano, C.-A. (4) 1343-1353

Lacosta, A.-M., see Pérez-Grijalba, V. (1) 47-56

Laitinen, M., see Martiskainen, H. (2) 565-573

Laks, J., see Sousa, M.F.B. (3) 905-913

Lamy, E., see Boutoleau-Bretonnière, C. (2) 625-630

Lan, Y.-L., J. Zhao and S. Li, Update on the Neuroprotective Effect of Estrogen Receptor Alpha Against Alzheimer's Disease (4) 1137-1148

Lange, K., see Schmidt, C. (4) 1229-1236

Lannfelt, L., see Tucker, S. (2) 575-588

Laterza, O.F., see Zhao, X. (2) 549-563

Laudon, H., see Tucker, S. (2) 575-588

Lautenschlager, N., see Hollands, S. (2) 677-686

Lavecchia, A.M., see Bedse, G. (4) 1115-1136

Lechner, A., see Benke, T. (2) 443-449

Lee, H.-g., see Hradek, A.C. (1) 57-65

Lee, H.-P., see Hradek, A.C. (1) 57-65

Lee, J., see Song, H. (3) 797-807

Lee, J.-H., see Ye, B.S. (1) 143-152

Lee, J.-S., see Ye, B.S. (1) 143-152

Lee, Y., see Ye, B.S. (1) 143-152 
Lehtimäki, T., see Martiskainen, H. (2) 565-573

Leinonen, V., see Martiskainen, H. (2) 565-573

Lejavova, K., K. Ondicova, L. Horvathova, N. Hegedusova, V. Cubinkova, P. Vargovic, G. Manz, P. Filipcik, B. Mravec, M. Novak and R. Kvetnansky, Stress-Induced Activation of the Sympathoadrenal System is Determined by Genetic Background in Rat Models of Tauopathy (4) $1157-1161$

Lejnine, S., see Zhao, X. (2) 549-563

Lelental, N., see Lewczuk, P. (1) 183-191

Lerner, Y., see Oren, N. (1) 19-22

Lewczuk, P., N. Lelental, P. Spitzer, J.M. Maler and J. Kornhuber, Amyloid- $\beta$ 42/40 Cerebrospinal Fluid Concentration Ratio in the Diagnostics of Alzheimer's Disease: Validation of Two Novel Assays (1) 183-191

Lewczuk, P., see Mroczko, B. (3) 1031-1037

Lhommel, R., see Ivanoiu, A. (3) 835-847

Li, C., see Chen, T. (1) 133-142

Li, H., see Zhao, H. (3) 1017-1030

Li, J., see Wang, D. (2) 519-534

Li, J., see Wang, D. (2) 519-534

Li, J., see Wang, Y. (2) 451-463

Li, J.-c., L. Han, Y.-x. Wen, Y.-x. Yang, S. Li, X.-s. Li, C.-j. Zhao, T.-y. Wang, H. Chen, Y. Liu, C.-1. Qi, X.-d. He, Q.-l. Gu, Y.-x. Ye, Y. Zhang, R. Huang, Y.-e Wu, R.-r. He, H. Kurihara, X.-y. Song, L. Cao and L.-j. Wang, Increased Permeability of the Blood-Brain Barrier and Alzheimer's DiseaseLike Alterations in Slit-2 Transgenic Mice (2) 535-548

Li, J.-G. and D. Praticò, High Levels of Homocysteine Results in Cerebral Amyloid Angiopathy in Mice (1) 29-35

Li, J.-j., see Tan, J.-p. (4) 1403-1412

Li, L., see Wang, D. (2) 519-534

Li, M., see Zhang, B. (2) 687-697

Li, N., see Tan, J.-p. (4) 1403-1412

Li, P., see Wang, J. (2) 465-477

Li, S., see Lan, Y.-L. (4) 1137-1148

Li, S., see Li, J.-c. (2) 535-548

Li, S., see Peng, L. (1) 227-242

Li, S., see Wang, D. (2) 519-534

Li, X.-H., see Du, L.-L. (3) 775-784

Li, X.-s., see Li, J.-c. (2) 535-548

Li, Y., see Wang, D. (2) 519-534

Li, Y.-F., see Zeng, F. (2) 511-518

Lian, Y., see Zeng, F. (2) 511-518

Liddle, J., see Pachana, N.A. (3) 701-707

Lim, C.S., see Khan, T.K. (2) 491-509
Lim, Y.Y., see Hollands, S. (2) 677-686

Lind, G., see Ferreira, D. (3) 1059-1072

Linderoth, B., see Ferreira, D. (3) 1059-1072

Lingler, J.H., Investigators' Perceptions of Research Oversight: Are Regulators Really Undermining Autonomy? (3) 709-710

Lirani-Silva, E., see Simieli, L. (2) 435-441

Liu, D., see Chen, J. (2) 657-668

Liu, D., see Gifford, K.A. (1) 121-132

Liu, J., see Ma, Q. (4) 1413-1427

Liu, J., see Peng, L. (1) 227-242

Liu, L.-B., see Du, L.-L. (3) 775-784

Liu, R., see Du, L.-L. (3) 775-784

Liu, R., see Wang, X.-L. (1) 153-165

Liu, Y., see Li, J.-c. (2) 535-548

Liu-Seifert, H., E. Siemers, K. Sundell, K. Price, B. Han, K. Selzler, P. Aisen, J. Cummings, J. Raskin and R. Mohs, Cognitive and Functional Decline and Their Relationship in Patients with Mild Alzheimer's Dementia (3) 949-955

Llanero-Luque, M., see Cuesta, P. (1) 259-273

Logsdon, A.F., see Lucke-Wold, B.P. (3) 711-724

López, M.E., see Cuesta, P. (1) 259-273

Lopez, O.L., see Wang, X. (2) 649-655

Lord, A., see Tucker, S. (2) 575-588

Lowe, D.A., see Balsis, S. (1) 289-290

Lu, F., see Zhang, B. (2) 687-697

Lu, Z., see Gifford, K.A. (1) 121-132

Lucke-Wold, B.P., R.C. Turner, A.F. Logsdon, J.W. Simpkins, D.L. Alkon, K.E. Smith, Y.-W. Chen, Z. Tan, J.D. Huber and C.L. Rosen, Common Mechanisms of Alzheimer's Disease and Ischemic Stroke: The Role of Protein Kinase $\mathrm{C}$ in the Progression of Age-Related Neurodegeneration (3) 711-724

Luo, H.-B., see Zeng, F. (2) 511-518

Luo, S., see Wang, D. (2) 519-534

Ma, F., T. Wu, R. Miao, Y. yu Xiao, W. Zhang and G. Huang, Conversion of Mild Cognitive Impairment to Dementia among Subjects with Diabetes: A Population-Based Study of Incidence and Risk Factors with Five Years of Follow-up (4) 14411449

Ma, N., see Wang, D. (2) 519-534

Ma, Q., M. Ying, X. Sui, H. Zhang, H. Huang, L. Yang, X. Huang, Z. Zhuang, J. Liu and X. Yang, Chronic Copper Exposure Causes Spatial Memory Impairment, Selective Loss of Hippocampal Synaptic Proteins, and Activation of PKR/eIF2 $\alpha$ Pathway in Mice (4) 1413-1427 
Mackin, R.S., P. Insel, J. Zhang, B. Mohlenhoff, D. Galasko, M. Weiner, N. Mattsson and for the Alzheimer's Disease Neuroimaging Initiative, Cerebrospinal Fluid $\alpha$-Synuclein and Lewy Body-Like Symptoms in Normal Controls, Mild Cognitive Impairment, and Alzheimer's Disease (3) 1007-1016

Maderna, E., C. Cattaneo, F. Cacciatore, M. Catania, G.D. Fede, F. Tagliavini and G. Giaccone, Divergent Cognitive Status with the Same Braak Stage of Neurofibrillary Pathology: Does the Pattern of Amyloid- $\beta$ Deposits Make the Difference? (2) 375-379

Maestú, F., see Cuesta, P. (1) 259-273

Magnani, G., see Cerami, C. (2) 385-395

Magnani, G., see Santangelo, R. (4) 1429-1440

Maheshwari, P. and G.D. Eslick, Bacterial Infection and Alzheimer's Disease: A Meta-Analysis (3) 957-966

Maier, W.C., see Takizawa, C. (4) 1271-1284

Majerova, P., see Paholikova, K. (3) 915-926

Mäkinen, P., see Martiskainen, H. (2) 565-573

Maldonado, R., see Aso, E. (3) 977-991

Maler, J.M., see Lewczuk, P. (1) 183-191

Mandusic, V., see Bajic, V. (4) 1251-1259

Manz, G., see Lejavova, K. (4) 1157-1161

Marcone, A., see Cerami, C. (2) 385-395

Marcos, A., see Cuesta, P. (1) 259-273

Marder, K.S., see Seifan, A. (1) 315-324

Mari, D., see Serpente, M. (3) 757-761

Mariani, C., see Serpente, M. (3) 757-761

Mariani, E., see Gironi, M. (4) 1199-1213

Marín, R., see Díaz, M. (4) 1185-1198

Marksteiner, J., see Benke, T. (2) 443-449

Martin, B., see Gifford, K.A. (1) 121-132

Martín, V., see Díaz, M. (4) 1185-1198

Martínez-Aguirre, X., see Calderón-Garcidueñas, L. (3) $1039-1058$

Martinez-Ramirez, S., see Ni, J. (4) 1325-1330

Martins, R.N., see Hollands, S. (2) 677-686

Martiskainen, H., S. Helisalmi, J. Viswanathan, M. Kurki, A. Hall, S.-K. Herukka, T. Sarajärvi, T. Natunen, K.M.A. Kurkinen, J. Huovinen, P. Mäkinen, M. Laitinen, A.M. Koivisto, K.M. Mattila, T. Lehtimäki, A.M. Remes, V. Leinonen, A. Haapasalo, H. Soininen and M. Hiltunen, Effects of Alzheimer's Disease-Associated Risk Loci on Cerebrospinal Fluid Biomarkers and Disease Progression: A Polygenic Risk Score Approach (2) 565-573

Maruff, P., see Hollands, S. (2) 677-686

Masliah, E., see Campbell, S.N. (3) 967-976
Masters, C.L., see Hollands, S. (2) 677-686

Maté, I., J. Cruces, L. Giménez-Llort and M. De la Fuente, Function and Redox State of Peritoneal Leukocytes as Preclinical and Prodromic Markers in a Longitudinal Study of Triple-Transgenic Mice for Alzheimer's Disease (1) 213-226

Mattila, K.M., see Martiskainen, H. (2) 565-573

Mattsson, N., see Mackin, R.S. (3) 1007-1016

Mauleón, A., see Hernández, I. (1) 325-334

Mccarty, D.M., see Naughton, B.J. (1) 93-108

McEvoy, M., see Muenchhoff, J. (4) 1355-1373

McKnight, P.E., see Graybeal, J.J. (3) 823-834

McNeely, A.A., J. Ramirez, S.M. Nestor, J. Zhao, F. Gao, A. Kiss, D.T. Stuss and S.E. Black, Cholinergic Subcortical Hyperintensities in Alzheimer's Disease Patients from the Sunnybrook Dementia Study: Relationships with Cognitive Dysfunction and Hippocampal Atrophy (3) 785-796

McNeely, A.A., see Ramirez, J. (2) 415-424

Meadows, A.S., see Naughton, B.J. (1) 93-108

Medeiros, R., see Dunn, H.C. (3) 893-903

Melo, T., see Monteiro-Cardoso, V.F. (4) 1375-1392

Meng, X.-F., see Tan, M.-S. (2) 589-603

Meng, X.-F., see Zhou, J. (2) 479-489

Mez, J., see Seifan, A. (1) 315-324

Miao, R., see Ma, F. (4) 1441-1449

Michalowsky, B., see Eichler, T. (4) 1303-1311

Michikawa, M., see Jung, C.-G. (4) 1215-1228

Michikawa, M., see Uhm, K.-O. (1) 243-257

Misumi, S., see Jung, C.-G. (4) 1215-1228

Misumi, S., see Uhm, K.-O. (1) 243-257

Miura, Y., see Uhm, K.-O. (1) 243-257

Mograbi, D.C., see Sousa, M.F.B. (3) 905-913

Mohlenhoff, B., see Mackin, R.S. (3) 1007-1016

Mohs, R., see Liu-Seifert, H. (3) 949-955

Möller, C., see Tucker, S. (2) 575-588

Monsch, A.U., see Glodzik, L. (3) 939-947

Montañés, M., see Pérez-Grijalba, V. (1) 47-56

Monte, L., see Campbell, S.N. (3) 967-976

Monteiro-Cardoso, V.F., M.M. Oliveira, T. Melo, M.R.M. Domingues, P.I. Moreira, E. Ferreiro, F. Peixoto and R.A. Videira, Cardiolipin Profile Changes are Associated to the Early Synaptic Mitochondrial Dysfunction in Alzheimer's Disease (4) 1375-1392

Montero-Odasso, M., S.W. Muir-Hunter, A. OtengAmoako, K. Gopaul, A. Islam, M. Borrie, J. Wells and M. Speechley, Donepezil Improves Gait Performance in Older Adults with Mild Alzheimer's Disease: A Phase II Clinical Trial (1) 193-199

Mook-Jung, I., see Song, H. (3) 797-807 
Moon, M., see Song, H. (3) 797-807

Moon, S.Y., see Ye, B.S. (1) 143-152

Moreira, P.I., see Monteiro-Cardoso, V.F. (4) 13751392

Morgan, D., see Haghighi, M. (4) 1261-1270

Mravec, B., see Lejavova, K. (4) 1157-1161

Mroczko, B., M. Groblewska, M. Zboch, P. Muszyński, A. Zajkowska, R. Borawska, M. Szmitkowski, J. Kornhuber and P. Lewczuk, Evaluation of VisininLike Protein 1 Concentrations in the Cerebrospinal Fluid of Patients with Mild Cognitive Impairment as a Dynamic Biomarker of Alzheimer's Disease (3) 1031-1037

Muenchhoff, J., A. Poljak, F. Song, M. Raftery, H. Brodaty, M. Duncan, M. McEvoy, J. Attia, P.W. Schofield and P.S. Sachdev, Plasma Protein Profiling of Mild Cognitive Impairment and Alzheimer's Disease Across Two Independent Cohorts (4) 1355-1373

Mufson, E.J., see Scheff, S.W. (3) 1073-1090

Muir-Hunter, S.W., see Montero-Odasso, M. (1) 193 199

Muller, W.E., see Hagl, S. (3) 927-938

Muñoz, F.J., see Vicario-Orri, E. (4) 1097-1113

Murrey, D.A., see Naughton, B.J. (1) 93-108

Muszyński, P., see Mroczko, B. (3) 1031-1037

Na, D.L., see Ye, B.S. (1) 143-152

Nacmias, B., see Piaceri, I. (4) 1169-1173

Nakamura, A., see Cuesta, P. (1) 259-273

Narayan, V.A., see Tryputsen,V. (3) 809-821

Nascimento, C.M.C., J.R. Pereira, L. Pires de Andrade, M. Garuffi, C. Ayan, D.S. Kerr, L.L. Talib, M.R. Cominetti and F. Stella, Physical Exercise Improves Peripheral BDNF Levels and Cognitive Functions in Mild Cognitive Impairment Elderly with Different BDNF Val66Met Genotypes (1) 81-91

Natunen, T., see Martiskainen, H. (2) 565-573

Naudé, P.J.W., see Dekker, A.D. (3) 871-891

Naughton, B.J., F.J. Duncan, D.A. Murrey, A.S. Meadows, D.E. Newsom, N. Stoicea, P. White, D.W. Scharre, D.M. Mccarty and H. Fu, Blood Genome-Wide Transcriptional Profiles Reflect Broad Molecular Impairments and Strong BloodBrain Links in Alzheimer's Disease (1) 93-108

Nelson, T.J., see Khan, T.K. (2) 491-509

Nemni, R., see Gironi, M. (4) 1199-1213

Nestor, S.M., see McNeely, A.A. (3) 785-796

Newsom, D.E., see Naughton, B.J. (1) 93-108

Ni, J., E. Auriel, S. Martinez-Ramirez, B. Keil, A.K.
Reed, P. Fotiadis, E.M. Gurol, S.M. Greenberg and A. Viswanathan, Cortical Localization of Microbleeds in Cerebral Amyloid Angiopathy: An Ultra High-Field 7T MRI Study (4) 1325-1330

Noble, J.M., see Seifan, A. (1) 315-324

Nogueira, M.L., see Sousa, M.F.B. (3) 905-913

Nourhashemi, F., see Villars, H. (1) 167-176

Novak, G.P., see Tryputsen,V. (3) 809-821

Novak, M. see Lejavova, K. (4) 1157-1161

Novak, M., see Paholikova, K. (3) 915-926

Nugent, S., see Castellano, C.-A. (4) 1343-1353

Oliva-Moreno, J., see Peña-Longobardo, L.M. (4) 1293-1302

Oliveira, M.M., see Monteiro-Cardoso, V.F. (4) 13751392

Olsson, E., B. Karlström, L. Kilander, L. Byberg, T. Cederholm and P. Sjögren, Dietary Patterns and Cognitive Dysfunction in a 12-Year Follow-up Study of 70 Year Old Men (1) 109-119

Ondicova, K., see Lejavova, K. (4) 1157-1161

Opattova, A., see Paholikova, K. (3) 915-926

Opazo, C.M.,see Vicario-Orri, E. (4) 1097-1113

Orcioli-Silva, D., see Simieli, L. (2) 435-441

Oren, N., G. Yogev-Seligmann, E. Ash, T. Hendler, N. Giladi and Y. Lerner, The Montreal Cognitive Assessment in Cognitively-Intact Elderly: A Case for Age-adjusted Cutoffs (1) 19-22

Osswald, G., see Tucker, S. (2) 575-588

Oteng-Amoako, A., see Montero-Odasso, M. (1) 193199

Pachana, N.A., J. Liddle, N.M. Peel, E. Beattie, C. Juang and B.G. Knight, Can We Do Better? Researchers' Experiences with Ethical Review Boards on Projects with Later Life as a Focus (3) 701-707

Paholikova, K., B. Salingova, A. Opattova, R. Skrabana, P. Majerova, N. Zilka, B. Kovacech, M. Zilkova, P. Barath and M. Novak, N-terminal Truncation of Microtubule Associated Protein Tau Dysregulates its Cellular Localization (3) 915-926

Pallardy, A., see Boutoleau-Bretonnière, C. (2) 625-630

Palmer, R.F., see Bishnoi, R.J. (1) 37-45

Palmer, R.F., see Royall, D.R. (1) 275-287

Pang, T., see Wang, J. (2) 465-477

Pantoni, L., see Salvadori, E. (4) 1313-1323

Panza, F., see Solfrizzi, V. (2) 605-611

Papageorgiou, S.G., see Safouris, A. (1) 23-27

Paquet, N., see Castellano, C.-A. (4) 1343-1353

Park, K.W., see Ye, B.S. (1) 143-152 
Park, S.A., see Ye, B.S. (1) 143-152

Park, S.-B., see Calderón-Garcidueñas, L. (3) 10391058

Park, S.K., see Song, H. (3) 797-807

Parkin, E.T., see Gough, M. (4) 1163-1168

Passerini, G., see Santangelo, R. (4) 1429-1440

Paulo de Magalhães Oliveira Júnior, P., see Andrade de Oliveira, A. (1) 201-212

Paxinos, G., see Fu, Y.H. (3) 857-869

Peel, N.M., see Pachana, N.A. (3) 701-707

Peixoto, F., see Monteiro-Cardoso, V.F. (4) 1375-1392

Peña-Longobardo, L.M. and J. Oliva-Moreno, Caregiver Burden in Alzheimer's Disease Patients in Spain (4) 1293-1302

Peng, L., Y. Yu, J. Liu, S. Li, H. He, N. Cheng and R.D. Ye, The Chemerin Receptor CMKLR1 is a Functional Receptor for Amyloid- $\beta$ Peptide (1) 227-242

Peng, Y., see Wang, D. (2) 519-534

Perani, D., see Cerami, C. (2) 385-395

Pereira, J.R., see Nascimento, C.M.C. (1) 81-91

Pereiro, A.X., see Cespón, J. (2) 631-647

Perez de Lara, M.J. and J. Pintor, Presence and Release of ATP from the Retina in an Alzheimer's Disease Model (1) 177-181

Pérez-Grijalba, V., P. Pesini, J.A. Allué, L. Sarasa, M. Montañés, A.-M. Lacosta, D. Casabona, I. San-José, M. Boada, L. Tárraga, A. Ruiz and M. Sarasa, $A \beta_{1-17}$ is a Major Amyloid- $\beta$ Fragment Isoform in Cerebrospinal Fluid and Blood with Possible Diagnostic Value in Alzheimer's Disease (1) $47-56$

Perrar, K.M., H. Schmidt, Y. Eisenmann, B. Cremer and R. Voltz, Needs of People with Severe Dementia at the End-of-Life: A Systematic Review (2) 397413

Perrin, A., see Villars, H. (1) 167-176

Pesini, P., see Pérez-Grijalba, V. (1) 47-56

Peter, C., see Schmidt, C. (4) 1229-1236

Piaceri, I., B. Raspanti, A. Tedde, S. Bagnoli, S. Sorbi and B. Nacmias, Epigenetic Modifications in Alzheimer's Disease: Cause or Effect? (4) 11691173

Pietrzak, R.H., see Hollands, S. (2) 677-686

Pinto, P., see Santangelo, R. (4) 1429-1440

Pintor, J., see Perez de Lara, M.J. (1) 177-181

Pires de Andrade, L., see Nascimento, C.M.C. (1) 81-91

Pisa, D., R. Alonso, A. Juarranz, A. Rábano and L. Carrasco, Direct Visualization of Fungal Infection in Brains from Patients with Alzheimer's Disease (2) 613-624

Poggesi, A., see Salvadori, E. (4) 1313-1323
Poljak, A., see Muenchhoff, J. (4) 1355-1373

Poole, S., S.K. Singhrao, S. Chukkapalli, M. Rivera, I. Velsko, L. Kesavalu and S. Crean, Active Invasion of Porphyromonas gingivalis and InfectionInduced Complement Activation in $\mathrm{ApoE}^{-/-}$Mice Brains (1) 67-80

Pracucci, G., see Salvadori, E. (4) 1313-1323

Praticò, D., see Li, J.-G. (1) 29-35

Price, D.A., see Scheff, S.W. (3) 1073-1090

Price, K., see Liu-Seifert, H. (3) 949-955

Qi, C.-1., see Li, J.-c. (2) 535-548

Qi, L.-W., see Wang, J. (2) 465-477

Qian, L., see Zhang, B. (2) 687-697

Qiu, M., see Wang, X.-L. (1) 153-165

Quenon, L., see Ivanoiu, A. (3) 835-847

Rábano, A., see Pisa, D. (2) 613-624

Raftery, M., see Muenchhoff, J. (4) 1355-1373

Raghavan, N., see Tryputsen,V. (3) 809-821

Rahardjo, T.B., see Xu, X. (2) 669-675

Ramani, K., see Kumar, P. (2) 341-361

Ramaraj, T.C., see Zhao, X. (2) 549-563

Ramirez, J., C. Berezuk, A.A. McNeely, C.J.M. Scott, F. Gao and S.E. Black, Visible Virchow-Robin Spaces on Magnetic Resonance Imaging of Alzheimer's Disease Patients and Normal Elderly from the Sunnybrook Dementia Study (2) 415-424

Ramirez, J., see McNeely, A.A. (3) 785-796

Ransmayr, G., see Benke, T. (2) 443-449

Raskin, J., see Liu-Seifert, H. (3) 949-955

Raspanti, B., see Piaceri, I. (4) 1169-1173

Reed, A.K., see Ni, J. (4) 1325-1330

Remes, A.M., see Martiskainen, H. (2) 565-573

Rice, K.C., see Campbell, S.N. (3) 967-976

Rissman, R.A., see Campbell, S.N. (3) 967-976

Rivera, M., see Poole, S. (1) 67-80

Roberts, K.N., see Scheff, S.W. (3) 1073-1090

Rodrigues, A.J., see Sotiropoulos, I. (3) 763-774

Roe, A.D., see Campbell, S.N. (3) 967-976

Romano, A., see Bedse, G. (4) 1115-1136

Romano, R., see Gifford, K.A. (1) 121-132

Rosa, P.A.D., see Cerami, C. (2) 385-395

Rosa, R.D.L., see Sousa, M.F.B. (3) 905-913

Rosen, C.L., see Lucke-Wold, B.P. (3) 711-724

Rosende-Roca, M., see Hernández, I. (1) 325-334

Rotondo, E., see Serpente, M. (3) 757-761

Rowe, C.C., see Hollands, S. (2) 677-686

Royall, D.R., R.F. Palmer and for the Texas Alzheimer's Research and Care Consortium, Ethnicity Moderates Dementia's Biomarkers (1) 275-287

Royall, D.R., see Bishnoi, R.J. (1) 37-45 
Ruberu, K., see Zhao, H. (3) 1017-1030

Ruiz, A., see Hernández, I. (1) 325-334

Ruiz, A., see Pérez-Grijalba, V. (1) 47-56

Rusinek, H., see Glodzik, L. (3) 939-947

Saad, Y., D. Segal and A. Ayali, Enhanced Neurite Outgrowth and Branching Precede Increased Amyloid- $\beta$-Induced Neuronal Apoptosis in a Novel Alzheimer's Disease Model (3) 993-1006

Sachdev, P.S., see Muenchhoff, J. (4) 1355-1373

Safouris, A., A.-S. Hambye, C. Sculier, S.G. Papageorgiou, S.N. Vasdekis, M.-D. Gazagnes and G. Tsivgoulis, Chronic Brain Hypoperfusion due to Multi-Vessel Extracranial Atherosclerotic Disease: A Potentially Reversible Cause of Cognitive Impairment (1) 23-27

Sahlin, C., see Tucker, S. (2) 575-588

Salingova, B., see Paholikova, K. (3) 915-926

Salvadori, E., A. Poggesi, G. Pracucci, D. Inzitari, L. Pantoni and on behalf of the VMCI-Tuscany Study Group, Development and Psychometric Properties of a Neuropsychological Battery for Mild Cognitive Impairment with Small Vessel Disease: The VMCI-Tuscany Study (4) 1313-1323

Samtani, M., see Tryputsen,V. (3) 809-821

Sánchez-Pla, A., see Aso, E. (3) 977-991

Sancho, M., see Cuesta, P. (1) 259-273

Sanin, G., see Benke, T. (2) 443-449

San-José, I., see Pérez-Grijalba, V. (1) 47-56

Santangelo, R., E. Coppi, L. Ferrari, M.P. Bernasconi, P. Pinto, G. Passerini, G. Comi and G. Magnani, Cerebrospinal Fluid Biomarkers Can Play a Pivotal Role in the Diagnostic Work Up of Primary Progressive Aphasia (4) 1429-1440

Santos, R.L., see Sousa, M.F.B. (3) 905-913

Sarajärvi, T., see Martiskainen, H. (2) 565-573

Sarasa, L., see Pérez-Grijalba, V. (1) 47-56

Sarasa, M., see Pérez-Grijalba, V. (1) 47-56

Sarathi-Mukherjee, P., see Calderón-Garcidueñas, L. (3) $1039-1058$

Satlin, A., see Tucker, S. (2) 575-588

Sato, J.R., see Andrade de Oliveira, A. (1) 201-212

Sattlecker, M., see Chiam, J.T.W. (1) 303-314

Scarpini, E., see Serpente, M. (3) 757-761

Scharre, D.W., see Naughton, B.J. (1) 93-108

Scheff, S.W., D.A. Price, M.A. Ansari, K.N. Roberts, F.A. Schmitt, M.D. Ikonomovic and E.J. Mufson, Synaptic Change in the Posterior Cingulate Gyrus in the Progression of Alzheimer's Disease (3) 1073-1090

Schmidt, C., N. Gerlach, C. Peter, K. Gherib, K. Lange, T. Friede and I. Zerr, Cerebrospinal Fluid
Apolipoprotein E Concentration and Progression of Alzheimer's Disease (4) 1229-1236

Schmidt, H., see Perrar, K.M. (2) 397-413

Schmidt, R., see Benke, T. (2) 443-449

Schmitt, F.A., see Scheff, S.W. (3) 1073-1090

Schofield, P.W., see Muenchhoff, J. (4) 1355-1373

Schreiner, B., L. Hedskog, B. Wiehager and M. Ankarcrona, Amyloid- $\beta$ Peptides are Generated in Mitochondria-Associated Endoplasmic Reticulum Membranes (2) 369-374

Schultzberg, M., see Zhu, M. (4) 1237-1250

Schwarz, C.G., see Zhang, B. (2) 687-697

Scott, C.J.M., see Ramirez, J. (2) 415-424

Sculier, C., see Safouris, A. (1) 23-27

Segal, D., see Saad, Y. (3) 993-1006

Seifan, A., K.S. Marder, J. Mez, J.M. Noble, E.P. Cortes, J.P. Vonsattel and L.S. Honig, Hippocampal Laminar Distribution of Tau Relates to Alzheimer's Disease and Age of Onset (1) 315-324

Seiger, Å., see Ferreira, D. (3) 1059-1072

Selzler, K., see Liu-Seifert, H. (3) 949-955

Sen, A., see Khan, T.K. (2) 491-509

Seo, S.W., see Ye, B.S. (1) 143-152

Serpente, M., C. Fenoglio, F. Clerici, R. Bonsi, B. Arosio, S.M.G. Cioffi, E. Rotondo, M. Franceschi, F.M. Boneschi, D. Mari, C. Mariani, E. Scarpini and D. Galimberti, Transmembrane Protein 106B Gene (TMEM106B) Variability and Influence on Progranulin Plasma Levels in Patients with Alzheimer's Disease (3) 757-761

Serrano-Heranz, R., see Ascensión Zea-Sevilla, M. (2) 363-367

Shakespeare, T.J., K.X.X. Yong, D. Foxe, J. Hodges and S.J. Crutch, Pronounced Impairment of Everyday Skills and Self-Care in Posterior Cortical Atrophy (2) 381-384

Shi, X.-Q., see Zeng, F. (2) 511-518

Shi, Y., see Chen, J. (2) 657-668

Shi, Z.-Q., see Wang, J. (2) 465-477

Shoemark, D.K. and S.J. Allen, The Microbiome and Disease: Reviewing the Links between the Oral Microbiome, Aging, and Alzheimer's Disease (3) 725-738

Shu, H., see Chen, J. (2) 657-668

Siedlak, S.L., see Hradek, A.C. (1) 57-65

Siemers, E., see Liu-Seifert, H. (3) 949-955

Silva, J., see Sotiropoulos, I. (3) 763-774

Simieli, L., F.A. Barbieri, D. Orcioli-Silva, E. LiraniSilva, F. Stella and L.T.B. Gobbi, Obstacle Crossing with Dual Tasking is a Danger for Individuals with Alzheimer's Disease and for Healthy Older People (2) 435-441 
Simmons, A., see Ferreira, D. (3) 1059-1072

Simões, P., see Sousa, M.F.B. (3) 905-913

Simpkins, J.W., see Lucke-Wold, B.P. (3) 711-724

Singhrao, S.K., see Poole, S. (1) 67-80

Sjödahl, J., see Tucker, S. (2) 575-588

Sjögren, P., see Olsson, E. (1) 109-119

Skrabana, R., see Paholikova, K. (3) 915-926

Small, B., see Haghighi, M. (4) 1261-1270

Smith, A., see Haghighi, M. (4) 1261-1270

Smith, K.E., see Lucke-Wold, B.P. (3) 711-724

Snyder, P.J., see Hollands, S. (2) 677-686

Söderberg, L., see Tucker, S. (2) 575-588

Soininen, H., see Martiskainen, H. (2) 565-573

Solé-Casals, J., see Gallego-Jutglà, E. (4) 1175-1184

Solfrizzi, V. and F. Panza, Plant-Based Nutraceutical Interventions against Cognitive Impairment and Dementia: Meta-Analytic Evidence of Efficacy of a Standardized Gingko biloba Extract (2) 605-611

Sollberger, M., see Glodzik, L. (3) 939-947

Song, F., see Muenchhoff, J. (4) 1355-1373

Song, H., Y.J. Chang, M. Moon, S.K. Park, P.-T. Tran, V.-H. Hoang, J. Lee and I. Mook-Jung, Inhibition of Glutaminyl Cyclase Ameliorates Amyloid Pathology in an Animal Model of Alzheimer's Disease via the Modulation of $\gamma$-Secretase Activity (3) 797-807

Song, X.-y., see Li, J.-c. (2) 535-548

Sorbi, S., see Piaceri, I. (4) 1169-1173

Sotiropoulos, I., J. Silva, T. Kimura, A.J. Rodrigues, P. Costa, O.F.X. Almeida, N. Sousa and A. Takashima, Female Hippocampus Vulnerability to Environmental Stress, a Precipitating Factor in Tau Aggregation Pathology (3) 763-774

Sotolongo-Grau, O., see Hernández, I. (1) 325-334

Sousa, M.F.B., R.L. Santos, M.L. Nogueira, T. Belfort, R.D.L. Rosa, B. Torres, P. Simões, D.C. Mograbi, J. Laks and M.C.N. Dourado, Awareness of Disease is Different for Cognitive and Functional Aspects in Mild Alzheimer's Disease: A One-Year Observation Study (3) 905-913

Sousa, N., see Sotiropoulos, I. (3) 763-774

Speechley, M., see Montero-Odasso, M. (1) 193-199

Spens, E., see Tucker, S. (2) 575-588

Spitzer, P., see Lewczuk, P. (1) 183-191

Spond, J., see Zhao, X. (2) 549-563

Spremo-Potparevic, B., see Bajic, V. (4) 1251-1259

Ståhlbom, A.K., see Thordardottir, S. (4) 1393-1402

Stefanova, E., see Bajic, V. (4) 1251-1259

Stella, F., see Nascimento, C.M.C. (1) 81-91

Stella, F., see Simieli, L. (2) 435-441

Stoicea, N., see Naughton, B.J. (1) 93-108
Stuss, D.T., see McNeely, A.A. (3) 785-796

Subaiea, G.M., A.H. Ahmed, L.I. Adwan and N.H. Zawia, Reduction of Amyloid- $\beta$ Deposition and Attenuation of Memory Deficits by Tolfenamic Acid (2) 425-433

Sui, X., see Ma, Q. (4) 1413-1427

Sun, X.-Y., see Wang, X.-L. (1) 153-165

Sundell, K., see Liu-Seifert, H. (3) 949-955

Sweet, R.A., see Wang, X. (2) 649-655

Szmitkowski, M., see Mroczko, B. (3) 1031-1037

Taché, Y., see Campbell, S.N. (3) 967-976

Tagliavini, F., see Maderna, E. (2) 375-379

Takashima, A., see Sotiropoulos, I. (3) 763-774

Takizawa, C., P.L. Thompson, A. van Walsem, C. Faure and W.C. Maier, Epidemiological and Economic Burden of Alzheimer's Disease: A Systematic Literature Review of Data across Europe and the United States of America (4) 1271-1284

Talib, L.L., see Nascimento, C.M.C. (1) 81-91

Tan, C.-C., see Tan, M.-S. (2) 589-603

Tan, C.-C., see Zhou, J. (2) 479-489

Tan, J.-p., N. Li, J. Gao, L.-n. Wang, Y.-m. Zhao, B.c. Yu, W.Du, W.-j. Zhang, L.-q.Cui, Q.-s. Wang, J.-j. Li, J.-s. Yang, J.-m. Yu, X.-n. Xia and P.-y. Zhou, Optimal Cutoff Scores for Dementia and Mild Cognitive Impairment of the Montreal Cognitive Assessment among Elderly and OldestOld Chinese Population (4) 1403-1412

Tan, L., see Tan, M.-S. (2) 589-603

Tan, L., see Zhou, J. (2) 479-489

Tan, M.-S., J.-T. Yu, C.-C. Tan, H.-F. Wang, X.-F. Meng, C. Wang, T. Jiang, X.-C. Zhu and L. Tan, Efficacy and Adverse Effects of Ginkgo Biloba for Cognitive Impairment and Dementia: A Systematic Review and Meta-Analysis (2) 589-603

Tan, Z., see Lucke-Wold, B.P. (3) 711-724

Tang, L., see Wang, D. (2) 519-534

Tao, B.-B., see Yu, D. (1) 291-302

Tárraga, L., see Hernández, I. (1) 325-334

Tárraga, L., see Pérez-Grijalba, V. (1) 47-56

Tedde, A., see Piaceri, I. (4) 1169-1173

Tegerstedt, K., see Tucker, S. (2) 575-588

Teipel, S., see Eichler, T. (4) 1303-1311

Thompson, P.L., see Takizawa, C. (4) 1271-1284

Thordardottir, S., A.K. Ståhlbom, D. Ferreira, O. Almkvist, E. Westman, H. Zetterberg, M. Eriksdotter, K. Blennow and C. Graff, Preclinical Cerebrospinal Fluid and Volumetric Magnetic Resonance Imaging Biomarkers in Swedish Familial Alzheimer's Disease (4) 1393-1402 
Thyrian, J.R., see Eichler, T. (4) 1303-1311

Tian, C., see Zhang, B. (2) 687-697

Torres, B., see Sousa, M.F.B. (3) 905-913

Torres, S.L., see Hradek, A.C. (1) 57-65

Torres-Jardón, R., see Calderón-Garcidueñas, L. 1039-1058

Tran, P.-T., see Song, H. (3) 797-807

Tremblay, S., see Castellano, C.-A. (4) 1343-1353

Tripodis, Y., see Gifford, K.A. (1) 121-132

Trojano, L., see Grossi, D. (3) 849-855

Tryputsen,V. , A. DiBernardo, M. Samtani, G.P. Novak, V.A. Narayan, N. Raghavan and the Alzheimer's Disease Neuroimaging Initiative, Optimizing Regions-of-Interest Composites for Capturing Treatment Effects on Brain Amyloid in Clinical Trials (3) 809-821

Tsivgoulis, G., see Safouris, A. (1) 23-27

Tucker, S., C. Möller, K. Tegerstedt, A. Lord, H. Laudon, J. Sjödahl, L. Söderberg, E. Spens, C. Sahlin, E.R. Waara, A. Satlin, P. Gellerfors, G. Osswald and L. Lannfelt, The Murine Version of BAN2401 (mAb158) Selectively Reduces Amyloid- $\beta$ Protofibrils in Brain and Cerebrospinal Fluid of tg-ArcSwe Mice (2) 575-588

Tuo, Q.-Z., see Wang, X.-L. (1) 153-165

Turcotte, É., see Castellano, C.-A. (4) 1343-1353

Turner, R.C., see Lucke-Wold, B.P. (3) 711-724

Tzouvaleka, E., see Bonoti, F. (4) 1285-1292

Ueda, Y., see Jung, C.-G. (4) 1215-1228

Uhm, K.-O., M.-J. Kim, M. Kawaguchi, H. Akatsu, Y. Miura, S. Misumi, H. Hida, E.-K. Choi, Y.-S. Kim, M. Michikawa and C.-G. Jung, ATBF1 is a Novel Amyloid- $\beta$ Protein Precursor (A $\beta$ PP) Binding Protein that Affects A $\beta P P$ Expression (1) 243-257

Uhm, K.-O., see Jung, C.-G. (4) 1215-1228

Uranüs, M., see Benke, T. (2) 443-449

van Duijn, C.M., see Dekker, A.D. (3) 871-891

van Walsem, A., see Takizawa, C. (4) 1271-1284

Vargas, L., see Hernández, I. (1) 325-334

Vargovic, P., see Lejavova, K. (4) 1157-1161

Vasdekis, S.N., see Safouris, A. (1) 23-27

Vegas-Lozano, E., see Aso, E. (3) 977-991

Vellas, B., see Villars, H. (1) 167-176

Velsko, I., see Poole, S. (1) 67-80

Vercelletto, M., see Boutoleau-Bretonnière, C. (2) 625630

Vermeiren, Y., see Dekker, A.D. (3) 871-891

Vialatte, F.-B., see Gallego-Jutglà, E. (4) 1175-1184

Vicario-Orri, E., C.M. Opazo and F.J. Muñoz,
The Pathophysiology of Axonal Transport in Alzheimer's Disease (4) 1097-1113

Videira, R.A., see Monteiro-Cardoso, V.F. (4) 13751392

Villars, H., C. Dupuy, A. Perrin, B. Vellas and F. Nourhashemi, Impact of a Therapeutic Educational Program on Quality of Life in Alzheimer's Disease: Results of a Pilot Study (1) 167-176

Villemagne, V.L., see Hollands, S. (2) 677-686

Viswanathan, A., see Ni, J. (4) 1325-1330

Viswanathan, J., see Martiskainen, H. (2) 565-573

Vlachos, F., see Bonoti, F. (4) 1285-1292

Vojdani, A., see Calderón-Garcidueñas, L. (3) 10391058

Voltz, R., see Perrar, K.M. (2) 397-413

Vonsattel, J.P., see Seifan, A. (1) 315-324

Waara, E.R., see Tucker, S. (2) 575-588

Wahlberg, L., see Ferreira, D. (3) 1059-1072

Wahlund, L.-O., see Ferreira, D. (3) 1059-1072

Wang, C., see Tan, M.-S. (2) 589-603

Wang, C., see Zhou, J. (2) 479-489

Wang, D., Y. Hui, Y. Peng, L. Tang, J. Jin, R. He, Y. Li, S. Zhang, L. Li, Y. Zhou, J. Li, N. Ma, J. Li, S. Li, X. Gao and S. Luo, Overexpression of Heme Oxygenase 1 Causes Cognitive Decline and Affects Pathways for Tauopathy in Mice (2) 519534

Wang, H.-F., see Tan, M.-S. (2) 589-603

Wang, H.-F., see Zhou, J. (2) 479-489

Wang, J., see Zhou, J. (2) 479-489

Wang, J., Z.-Q. Shi, M. Zhang, G.-Z. Xin, T. Pang, P. Zhou, J. Chen, L.-W. Qi, H. Yang, X. Xu and P. Li, Camptothecin and its Analogs Reduce Amyloid- $\beta$ Production and Amyloid- $\beta_{42}$-Induced IL-1 $\beta$ Production (2) 465-477

Wang, J.-Z., see Du, L.-L. (3) 775-784

Wang, J.-Z., see Wang, X.-L. (1) 153-165

Wang, K., see Zhang, B. (2) 687-697

Wang, L.-j., see Li, J.-c. (2) 535-548

Wang, L.-n., see Tan, J.-p. (4) 1403-1412

Wang, Q.-s., see Tan, J.-p. (4) 1403-1412

Wang, T.-y., see Li, J.-c. (2) 535-548

Wang, X., O.L. Lopez, R.A. Sweet, J.T. Becker, S.T. DeKosky, M.M. Barmada, F.Y. Demirci and M.I. Kamboh, Genetic Determinants of Disease Progression in Alzheimer's Disease (2) 649-655

Wang, X., see Zhu, M. (4) 1237-1250

Wang, X.-L., J. Zeng, Y. Yang, Y. Xiong, Z.-H. Zhang, M. Qiu, X. Yan, X.-Y. Sun, Q.-Z. Tuo, R. Liu and J.-Z. Wang, Helicobacter pylori Filtrate Induces 
Alzheimer-Like Tau Hyperphosphorylation by Activating Glycogen Synthase Kinase-3ß (1) 153165

Wang, Y., L. Wu, J. Li, D. Fang, C. Zhong, J.X. Chen and S.S. Yan, Synergistic Exacerbation of Mitochondrial and Synaptic Dysfunction and Resultant Learning and Memory Deficit in a Mouse Model of Diabetic Alzheimer's Disease (2) 451-463

Wang, Y.-J., see Zeng, F. (2) 511-518

Wang, Z., see Chen, J. (2) 657-668

Weiner, M., see Mackin, R.S. (3) 1007-1016

Weiner, R., see Zhao, X. (2) 549-563

Wells, J., see Montero-Odasso, M. (1) 193-199

Wen, Y.-X., see Li, J.-c. (2) 535-548

Westman, E., see Ferreira, D. (3) 1059-1072

Westman, E., see Thordardottir, S. (4) 1393-1402

White, P., see Naughton, B.J. (1) 93-108

Wiberg, M., see Ferreira, D. (3) 1059-1072

Wiehager, B., see Schreiner, B. (2) 369-374

Wu, C., see Chen, T. (1) 133-142

Wu, K., see Du, L.-L. (3) 775-784

Wu, L., see Wang, Y. (2) 451-463

Wu, T., see Ma, F. (4) 1441-1449

Wu, W., see Zhang, B. (2) 687-697

Wu, Y.-e, see Li, J.-c. (2) 535-548

Wucherer, D., see Eichler, T. (4) 1303-1311

Xia, X.-n., see Tan, J.-p. (4) 1403-1412

Xiao, S., see Xu, X. (2) 669-675

Xie, W.-T., see Zeng, F. (2) 511-518

Xin, G.-Z., see Wang, J. (2) 465-477

Xiong, Y., see Wang, X.-L. (1) 153-165

Xu, S., see Chen, T. (1) 133-142

$\mathrm{Xu}, \mathrm{X} ., \mathrm{S}$. Xiao, T.B. Rahardjo and E. Hogervorst, Tofu Intake is Associated with Poor Cognitive Performance among Community-Dwelling Elderly in China (2) 669-675

Xu, X., see Wang, J. (2) 465-477

Xu, Y., see Zhang, B. (2) 687-697

Yan, J.-K., see Yu, D. (1) 291-302

Yan, S.S., see Wang, Y. (2) 451-463

Yan, X., see Wang, X.-L. (1) 153-165

Yang, H., see Wang, J. (2) 465-477

Yang, J.-s., see Tan, J.-p. (4) 1403-1412

Yang, L., see Ma, Q. (4) 1413-1427

Yang, Q., see Yu, D. (1) 291-302

Yang, Q.X., see Zhang, B. (2) 687-697

Yang, S.-S., see Yu, D. (1) 291-302

Yang, X., see Ma, Q. (4) 1413-1427
Yang, Y., see Wang, X.-L. (1) 153-165

Yang, Y.-X., see Li, J.-c. (2) 535-548

Yang, Y.-Y., see Yu, D. (1) 291-302

Ye, B.S., J. Chin, S.Y. Kim, J.-S. Lee, E.-J. Kim, Y. Lee, C.H. Hong, S.H. Choi, K.W. Park, B.D. Ku, S.Y. Moon, S. Kim, S.-H. Han, J.-H. Lee, H.-K. Cheong, S.A. Park, J.H. Jeong, D.L. Na and S.W. Seo, The Heterogeneity and Natural History of Mild Cognitive Impairment of Visual Memory Predominant Type (1) 143-152

Ye, R.D., see Peng, L. (1) 227-242

Ye, Y.-x., see Li, J.-c. (2) 535-548

Yin, Z., see Zhang, B. (2) 687-697

Ying, M., see Ma, Q. (4) 1413-1427

Yogev-Seligmann, G., see Oren, N. (1) 19-22

Yong, K.X.X., see Shakespeare, T.J. (2) 381-384

yu Xiao, Y., see Ma, F. (4) 1441-1449

Yu, B.-c., see Tan, J.-p. (4) 1403-1412

Yu, D., B.-B. Tao, Y.-Y. Yang, L.-S. Du, S.-S. Yang, X.-J. He, Y.-W. Zhu, J.-K. Yan and Q. Yang, The IDO Inhibitor Coptisine Ameliorates Cognitive Impairment in a Mouse Model of Alzheimer's Disease (1) 291-302

Yu, H., see Zhang, B. (2) 687-697

Yu, J.-m., see Tan, J.-p. (4) 1403-1412

Yu, J.-T., see Tan, M.-S. (2) 589-603

Yu, J.-T., see Zhou, J. (2) 479-489

Yu, Y., see Peng, L. (1) 227-242

Zajkowska, A., see Mroczko, B. (3) 1031-1037

Zawia, N.H., see Subaiea, G.M. (2) 425-433

Zboch, M., see Mroczko, B. (3) 1031-1037

Zeng, F., W.-T. Xie, Y.-J. Wang, H.-B. Luo, X.-Q. Shi, H.-Q. Zou, Y.-Q. Zeng, Y.-F. Li, S.-R. Zhang and Y. Lian, General Public Perceptions and Attitudes toward Alzheimer's Disease from Five Cities in China (2) 511-518

Zeng, J., see Wang, X.-L. (1) 153-165

Zeng, Y.-Q., see Zeng, F. (2) 511-518

Zerr, I., see Schmidt, C. (4) 1229-1236

Zetterberg, H., see Thordardottir, S. (4) 1393-1402

Zhang, B., X. Zhang, F. Zhang, M. Li, C.G. Schwarz, J. Zhang, Z. Yin, L. Qian, H. Zhao, K. Wang, C. Tian, H. Yu, W. Chen, F. Lu, W. Wu, Q.X. Yang, Y. $\mathrm{Xu}$ and B. Zhu, Characterizing Topological Patterns in Amnestic Mild Cognitive Impairment by Quantitative Water Diffusivity (2) 687-697

Zhang, C., see Campbell, S.N. (3) 967-976

Zhang, C., see Zhao, X. (2) 549-563

Zhang, F., see Zhang, B. (2) 687-697

Zhang, F.-C., see Du, L.-L. (3) 775-784 
Zhang, H., see Ma, Q. (4) 1413-1427

Zhang, H.-B., see Du, L.-L. (3) 775-784

Zhang, J., see Mackin, R.S. (3) 1007-1016

Zhang, J., see Zhang, B. (2) 687-697

Zhang, M., see Wang, J. (2) 465-477

Zhang, S., see Wang, D. (2) 519-534

Zhang, S.-R., see Zeng, F. (2) 511-518

Zhang, W., see Ma, F. (4) 1441-1449

Zhang, W.-j., see Tan, J.-p. (4) 1403-1412

Zhang, X., see Chen, J. (2) 657-668

Zhang, X., see Zhang, B. (2) 687-697

Zhang, Y., see Li, J.-c. (2) 535-548

Zhang, Z., see Chen, J. (2) 657-668

Zhang, Z.-H., see Wang, X.-L. (1) 153-165

Zhao, C.-j., see Li, J.-c. (2) 535-548

Zhao, H., H. Li, K. Ruberu and B. Garner, Impaired Lysosomal Cobalamin Transport in Alzheimer's Disease (3) 1017-1030

Zhao, H., see Zhang, B. (2) 687-697

Zhao, J., see Lan, Y.-L. (4) 1137-1148

Zhao, J., see McNeely, A.A. (3) 785-796

Zhao, L.-N., see Du, L.-L. (3) 775-784

Zhao, X., S. Lejnine, J. Spond, C. Zhang, T.C. Ramaraj, D.J. Holder, H. Dai, R. Weiner and O.F. Laterza, A Candidate Plasma Protein Classifier to Identify Alzheimer's Disease (2) 549-563
Zhao, Y.-m., see Tan, J.-p. (4) 1403-1412

Zhong, C., see Wang, Y. (2) 451-463

Zhou, J., J.-T. Yu, H.-F. Wang, X.-F. Meng, C.-C. Tan, J. Wang, C. Wang and L. Tan, Association between Stroke and Alzheimer's Disease: Systematic Review and Meta-Analysis (2) 479-489

Zhou, P., see Wang, J. (2) 465-477

Zhou, P.-y., see Tan, J.-p. (4) 1403-1412

Zhou, X.-W., see Du, L.-L. (3) 775-784

Zhou, Y., see Wang, D. (2) 519-534

Zhu, B., see Zhang, B. (2) 687-697

Zhu, M., X. Wang, M. Schultzberg and E. Hjorth, Differential Regulation of Resolution in Inflammation induced by Amyloid- $\beta_{42}$ and Lipopolysaccharides in Human Microglia (4) 1237-1250

Zhu, X.-C., see Tan, M.-S. (2) 589-603

Zhu, Y.-W., see Yu, D. (1) 291-302

Zhuang, Z., see Ma, Q. (4) 1413-1427

Zilka, N., see Paholikova, K. (3) 915-926

Zilkova, M., see Paholikova, K. (3) 915-926

Zivkovic, L., see Bajic, V. (4) 1251-1259

Zou, H.-Q., see Zeng, F. (2) 511-518 